This document is a preprint version of the following article:

Title: Analyzing fluctuations in car-following

By: Wagner, Peter

Published in: Transportation Research Part B: Methodological Volume 46, Issue 10, December 2012, Pages 1384-1392

Publisher: Elsevier | Year: 2012

DOI: 10.1016/j.trb.2012.06.007

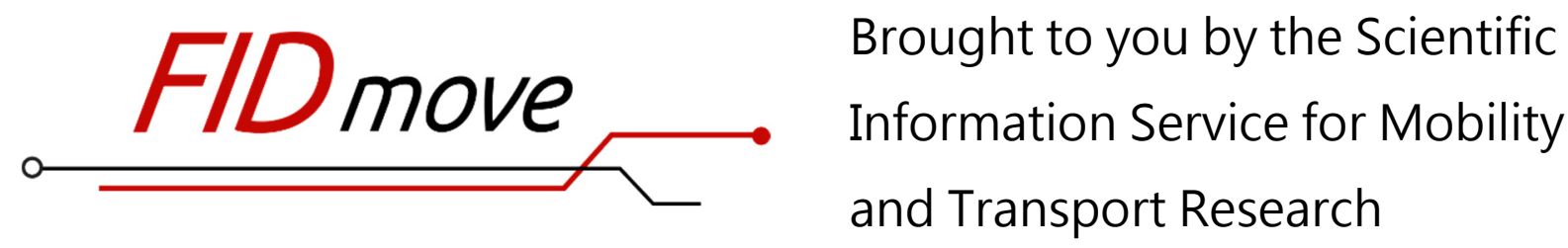

Fachinformationsdienst Mobilitäts- und Verkehrsforschung

Website: www.fid-move.de

Repository: publish.fid-move.de

Contact: publish@fid-move.de

(C) 2019. This manuscript version is made available under the CC-BYNC-ND 4.0 license.

http://creativecommons.org/licenses/by-nc-nd/4.0/ 


\title{
Analyzing fluctuations in car-following
}

\author{
Peter Wagner \\ Institute of Transport Systems, German Aerospace Centre, \\ Rutherfordstraße 2, 12489 Berlin, Germany, \\ e-Mail: peter.wagner@dlr.de, phone +493067055 237, fax +493067055291
}

\begin{abstract}
Many car-following models predict a stable car-following behavior with a very small fluctuation around an equilibrium value $g^{*}$ of the net headway $g$ with zero speed-difference $\Delta v$ between the following and the lead vehicle. However, it is well-known and additionally demonstrated by data in this paper, that the fluctuations are much larger than these models predict. Typically, the fluctuation in speed difference is around $\pm 2 \mathrm{~m} / \mathrm{s}$, while the fluctuation in the net time headway $T=g / v$ can be as big as one or even two seconds, which is as large as the mean time headway itself. By analyzing data from loop detectors as well as data from vehicle trajectories, evidence is provided that this randomness is not due to driver heterogeneity, but can be attributed to an internal stochasticity of the driver itself. A final model-based analysis supports the hypothesis, that the preferred headway of the driver is the parameter that is not kept constant but fluctuates strongly, thus causing the even macroscopically observable randomness in traffic flow.
\end{abstract}

Keywords: Time headway distribution, speed-difference distribution, stochasticity of traffic flow, driver heterogeneity, microscopic modeling, car-following

\section{Introduction}

The probability density functions (pdf's) of time-headway $p(T)$ and speed difference $p(\Delta v)$ between successive vehicles carry important information about the underlying processes that shape car-following (Krbalek et al., 2001), (Krbalek and Helbing, 2004). Although there is a lot of empirical and theoretical material on the time-headway pdf (Cowan, 1976), (May, 1990), (Hoogendoorn and Bovy, 1998) in car-following models these pdf's are rarely 
considered and used to test the predictions of these models. In the empirical analyses, usually only $p(T)$ is considered, while the pdf of speed differences $p(\Delta v)$ is mostly ignored. Here, it will be shown, that not only the time headway pdf, but also the speed difference pdf contain important information. Furthermore, the empirical analysis presented here can help to develop better car-following models that can not only describe the mean values, but also the fluctuations around these mean values correctly.

This paper will compare the fluctuation structure of single-vehicle stationary detector data (SSD-data) with trajectory data. In SSD-data, each record represents a different driver and therefore the whole data-set represents the maximum heterogeneity. This is different for trajectory data where each trajectory belongs to exactly one driver, therefore it reflects minimum heterogeneity. So, the question arises about the difference between these two sources of data when it comes to the analysis of the fluctuations (Ossen et al., 2007), (Kesting and Treiber, 2009). Here, the hypothesis will be tested that the fluctuation structure between these two types of data-sets should be different: the SSD-data should display much larger fluctuations since they stem from a large group of different drivers than do the data from the trajectories.

In many models, these fluctuations are not considered at all or are considered only as a kind of side-effect. If they are part of the model, then this is often modeled as acceleration noise. Acceleration noise is by construction rather limited in magnitude (since acceleration itself is) as well as in frequency: vehicles are heavy objects that do not change acceleration in short time intervals. Therefore, it is not very likely that the acceleration fluctuations are responsible for the width of the headway pdf.

\section{Data analysis}

This paper will draw mostly on two different data sources:

1. SSD-data are from a three-lane German freeway A3 (Knospe et al., 2002); the site was well away from any bottleneck or on- or off-ramps, so it represents undisturbed conditions.

2. Data from a field operational test (FOT) (Fancher et al., 1998) with more than 100 drivers. Each driver drove for a couple of weeks with an equipped car without driver assistant system, and another couple of weeks with a (simple) driver assistant system switched on. Only the data from driving without any assistance (the vehicles had also a speed 
cruise control) have been used here. The trajectories are sampled from a wide range of different driving conditions, from small urban roads to freeways.

Each record of the SSD-data contains a time stamp $t_{i}$, the speed $v_{i}$ and the length $l_{i}$ of the vehicle that crossed the detector, and the vehicle type. Each record of the data from the FOT contains more than 30 variables, of which in this analysis only the time stamp $t_{i}$, the speed $v_{i}$, the acceleration $a_{i}$, the throttle $\theta_{i}$, the distance $g_{i}$, and the speed-difference $\Delta v_{i}$ to the vehicle in front (supported by a binary flag that indicated that the distance sensor had found a valid target) have been used.

For both data-sets, the conditional pdf's of the speed-difference $p(\Delta v \mid v)$ and of the net time headways $p(T \mid v)$ will be analyzed. To ease notation, they will be written with speed $v$ as an index, i.e. $p_{v}(T)$. Using speed has the advantage that it can be measured easily, and it helps to compare different traffic situations. To a certain degree and with caution, even urban conditions can be compared to freeway conditions based on speed, as is the case for the FOT data - they stem from different roads. When using data from freeways, the speed serves as a simple proxy to the traffic density, with small speeds corresponding to high densities and large speeds corresponding to small densities.

For the SSD-data, the time-stamp relates to the head of the vehicle and therefore the net headway $T_{i}$ is computed as follows (with $v_{i}, l_{i}$ the speed and the length, respectively, of the $i$-th vehicle crossing the detector):

$$
T_{i}=t_{i}-t_{i-1}-\frac{l_{i-1}}{v_{i-1}} .
$$

and the speed difference is defined as:

$$
\Delta v_{i}=v_{i-1}-v_{i}
$$

To start, the pdf's of net headway and speed difference for the SSD-data will be analyzed first. The speeds are given in $\mathrm{km} / \mathrm{h}$ in the data and are 8 bit integer values $\{0, \ldots, 255\}$. They are converted to $\mathrm{m} / \mathrm{s}$, and then sorted into bins of width $1 \mathrm{~m} / \mathrm{s}$. For each value of the speed of the lead vehicle, a separate distribution will be sampled from the data.

Of course, the data still need some cleaning to eliminate inconsistencies, but not very much: more than $99.9 \%$ of the altogether 1'524 Mio data-points have been used in the subsequent analysis. 

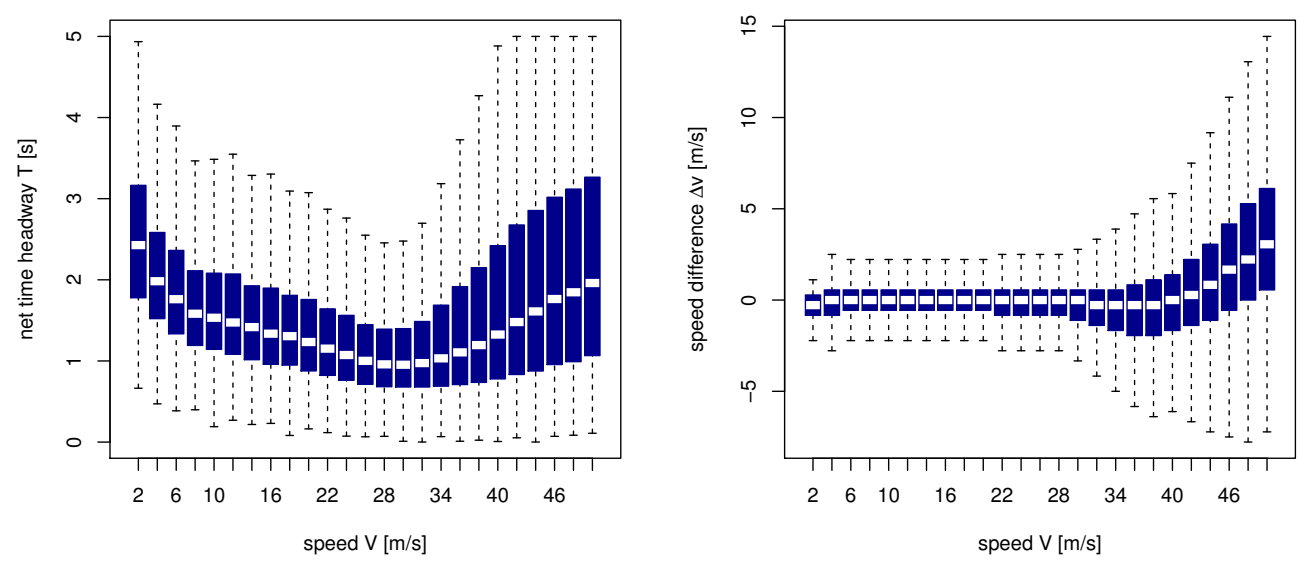

Figure 1: Box-and-Whisker plot of the two pdf's for the freeway data from the left lane only, showing the median and the 25 and 75 percentiles as function of the speed of the lead vehicle.

The result of this procedure is displayed in Figure 1 (left). For small speeds, the headway becomes ever larger, this is visible independent of the type of measurement (SSD-data as well as trajectory data). However, the loop detector data have a cut-off at $10 \mathrm{~km} / \mathrm{h}$ anyway, since those speeds are difficult to measure and the manufacturers of the detector believe these data to be not very precise.

As function of speed, the mean value of the headway gets smaller until it reaches a minimum value at a speed of around $v_{c} \approx 30 \mathrm{~m} / \mathrm{s}$; see (Wagner, 2004) for more examples of this behavior. Above this speed, both the mean value as well as the width of the headway pdf increases, since traffic now enters the free flow region, where vehicles do not interact with each other. This behavior is mimicked by the speed difference distribution (Figure 1, right), with the additional feature that for speeds in the congested region the speed distribution also changes its shape, this can be seen in Figure 2. It is a so called Laplace pdf for speeds below $v_{c}$, and it is roughly a normal pdf for large speeds. For large speeds, the normal pdf has a small rest of the Laplace pdf near $\Delta v=0$. This belongs to vehicles that closely follow another vehicle, which happens even in free traffic. The pdf of the Laplace 


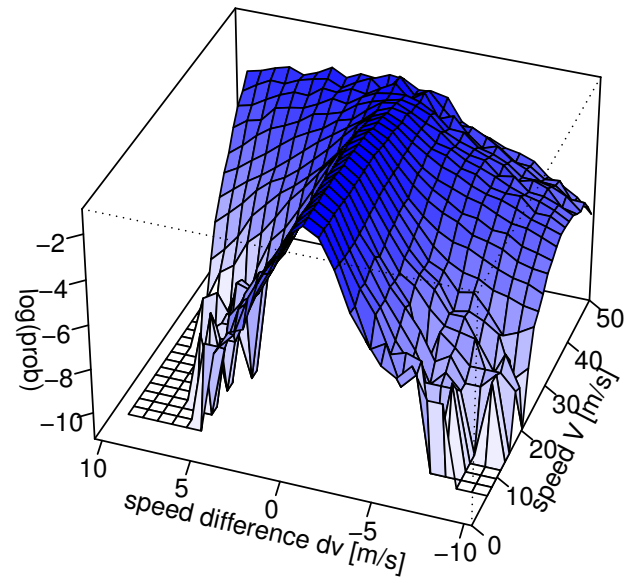

Figure 2: Pdf of the speed difference as function of the speed of the lead vehicle. The z-axis is logarithmic, so the change of form of the this pdf around $v_{c}$ can be clearly recognized.

distribution is defined as:

$$
p(\Delta v)=\frac{\beta}{2} \exp (-\beta|\Delta v|)
$$

So, the speed difference pdf very nicely separates the region where vehicles are interacting strongly with each other from the region where traffic is flowing freely. This behavior is not unique to the data analyzed here but has been found in other freeway data as well, such as the ones from the FSP (Petty, 1995) and the NGSIM project (Alexiadis et al., 2004). The precise value of $v_{c}$ is however not a universal constant but may depend on the site, the country, the speed-limit, the lane, and possible other external influences.

Note that Figure 1 and Figure 2 differ in another respect: while in Figure 2 all the data have been used, in Figure 1 only data with time headway smaller than $5 \mathrm{~s}$ have been sampled. This has been done to sample data preferentially from car-following situations. Unfortunately, that is no guarantee, that the data stem in fact from car-following episodes. 

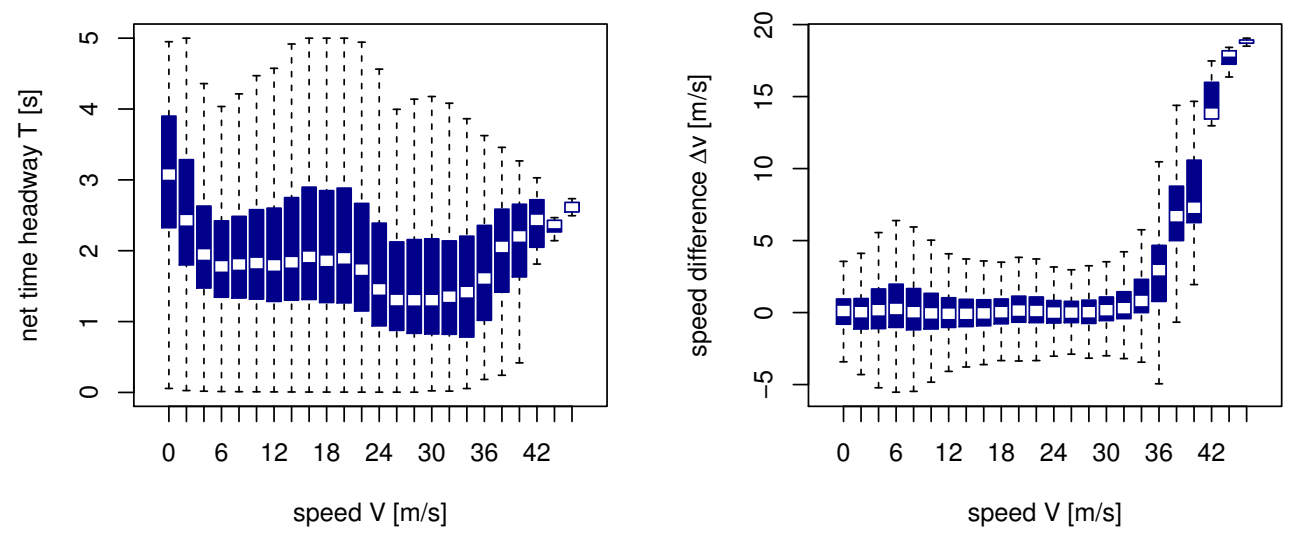

Figure 3: Box-and-Whisker plot of the two pdf's for the trajectory data, showing the median and the 25 and 75 percentiles as function of the speed of the lead vehicle.

In the following, the width of these pdf's is the main point of interest. As a measure for the width of the distributions, the difference between the $25 \%$ and the $75 \%$ percentile has been used (named $w_{50}(v)$ in the following), regarded again as a function of speed.

The same procedure has been performed on the trajectory data. However, in this case, more filtering is needed to deal with this more diverse data-set. The data contain an additional marker telling that a valid target vehicle in front has been established. However, this marker is always set to invalid for speeds below $13.3 \mathrm{~m} / \mathrm{s}$, so data below this speed have to be regarded with care. However, from a visual inspection of Figure 3 which has been generated including the "invalid" data (the corresponding data-sets nevertheless may contain a distance and a speed difference to the vehicle in front), nothing special could be seen in this area, so it might be concluded that these data are valid data despite the warning in the data-set.

It could be seen, that the trajectory data, although they are sampled in a more diverse environment, behave very similar to the data from the SSD. This holds true not only for the mean values, but also for the variance of the two variables under consideration. In Figure 4, this is demonstrated by overlaying all of the individual width versus speed curves for each driver with the curve from the SSD-data. This demonstrates, that there is only a small 

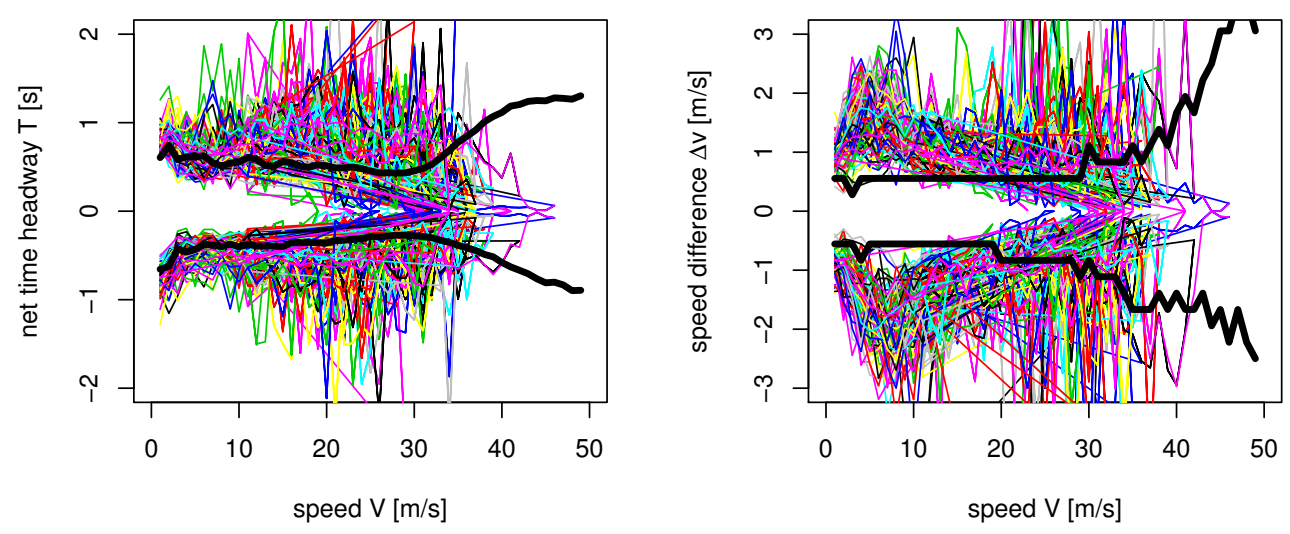

Figure 4: $25 \%$ and $75 \%$ percentile (median subtracted) for individual drivers (thin, colored lines) as well as for the SSD-data (thick black line). Left: net time headway, right: speed difference, again as function of the speed of the lead vehicle.

difference between the fluctuations displayed by a collection of heterogeneous drivers and an individual driver. This result is in line with results reported also in (Kesting and Treiber, 2009).

Therefore, the hypothesis can be formulated, that for the process of carfollowing the fluctuations especially in the headway are produced by an individual driver. The reminder of this paper tries to analyze this behavior into more detail.

Note, that similar patterns have been found also in other data-sets as well, e.g. in the NGSIM (Alexiadis et al., 2004) data, data from US freeways as well as in data from the other lanes of the German freeway A3 (Wagner, 2004). Sometimes, especially the median values display a more complicated behavior as function of speed, which may be due to a more heterogeneous vehicle composition - trucks are almost never found on the left lane whose results have been displayed in Figure 1.

\section{Some hypothesis regarding the size of the fluctuations}

When looking into trajectory data with a high data-quality, at least two apparent reasons for the fluctuations can be identified. Real drivers per- 
form a kind of noisy oscillation in the car-following space spanned by speeddifference and distance, and the amplitude of this oscillation corresponds directly to the empirically observed fluctuation widths. Moreover, the drivers do change their acceleration not in a smooth and continuous manner, but instead they change acceleration at so-called action-points in a jumpy fashion (Todosiev and Barbosa, 1963/64), (Wiedemann, 1974), (Wagner, 2006), (Wagner, 2005), (Hoogendoorn et al., 2012).

The trajectory data present a good opportunity to analyze the actionpoints in more detail. The throttle-state of the vehicle is measured as a value between 0 and $100 \%$, with a resolution of $0.5 \%$. The time resolution in the data is $0.1 \mathrm{~s}$. By counting the jumps between subsequent values of the throttle it turns out, that in more than $85 \%$ of the cases the value of the throttle does not change from one time-step to the next. The smallest value found is $67.9 \%$ (driver \# 104) and the largest value is $91.7 \%$ (driver \# 14). The time between real changes of the throttle is possibly Gamma distributed with a mean value of 0.4 to 0.7 seconds between subsequent action-points. Note, that this simple approach underestimates the time between real actionpoints, since it counts any arbitrarily small change in throttle as an actionpoint, while at least some of them do not constitute real and strong changes in throttle and therefore in acceleration. However, a precise analysis of the action-points is awkward (e.g. which change in throttle is needed to define an action-point) and not in the focus of this article.

For the oscillations itself, basically two mechanisms have been reported in the literature so far: they are either due to a finite reaction time of the driver which causes the car-following process to behave like an unstable harmonic oscillator, or they are created by a so called no-reaction-zone in the psychophysical models (Wiedemann, 1974). However, almost all of the models introduced in recent years do not follow anymore the reaction-time hypothesis and this for good reasons: drivers seem to anticipate the movement of the vehicle in front, thereby hiding any reaction time (for a nice empirical example, see e.g. (Wagner, 2006)). Reaction times possibly matter in the case of unexpected behavior of the lead vehicle, and this happens rarely. Models like the ones in (Gipps, 1981), (Krauss et al., 1997), or (Treiber et al., 2000 ) etc. have only a weak type of instability (if at all). This is due to an observed stability of traffic flow which is much more stable than most of the delay models can explain. However, this does not rule out that under certain circumstances, traffic flow can in fact be destabilized if an external perturbation is large enough. 
So, when looking at these models it can be seen, that they are stable and usually have very small fluctuations around the preferred distance even if they are extended by a stochastic acceleration term.

Therefore, it is useful to follow the assumption that it is the actionpoint mechanism that causes the oscillations to appear. However, this can be ruled out, too. By running for instance the model in (Treiber et al., 2000) (see the appendix for a numerical example) with an additional action point mechanism, the resulting fluctuations around the preferred headway still turned out to be small. While this argument seems to be supported only by a special car-following model, it can be made more general. It has been shown in (Wagner, 2011), that a normal micro-simulation model with any reasonable noise mechanism will yield in fact a rather small fluctuation around the preferred distance or it will produce an unstable model or it needs unrealistically large acceleration noise.

The basic idea in (Wagner, 2011) is to expand any car-following model with a preferred distance into a linear model (Helly, 1959), (Newell, 2002), (Flötteröd et al., 2011):

$$
\dot{v}=f(g, \Delta v, v) \approx \partial_{g} f\left(g-g^{*}\right)+\partial_{\Delta v} f \Delta v+\partial_{v} f\left(v-v^{*}\right)+D \xi .
$$

Endowed with an action-point mechanism and with an explicit acceleration noise, for such a model a stochastic discrete map for the speed-difference and distance to the vehicle in front can be derived. Since this is a stochastic linear dynamical system, the standard-deviation of the distance and speed fluctuation can be computed analytically. From this approach it could be concluded that the fluctuation size is in fact fairly small for most realistic parameter settings.

So, if reaction time or action points are not responsible for the large fluctuations in the headway, what is then causing them? In the following it is hypothesized, that the width of the headway distribution is due to a mechanism in the driver that changes the preferred headway in short time intervals. The most extreme of such an assumption is that the driver changes the preferred headway at each action point: there, not only the acceleration, but also the preferred headway changes. This is due to the fact, that the precise value of the distance is not very important to the driver, and this is what has been built into the three-phase models (Kerner, 2004): that the drivers have a large region of preferred headway values to choose from. Different from three-phase theory, it is hypothesized here that drivers do change this headway value almost all the time. 


\section{A model-based analysis}

To make progress, a resort has been made to a model-based analysis. As has been described already, most car-following models can be linearized around the preferred distance or even almost any other reasonable working point. To this end, the same Taylor expansion as in equation (3) to first order around the respective working points $\left(g^{*}, \Delta v^{*}=0, v^{*}\right)$ of the model is performed:

$$
\dot{v}=f(g, \Delta v, v) \approx \omega^{2}\left(g-g^{*}\right)+\gamma \Delta v+\alpha\left(v-v^{*}\right) .
$$

In this equation, the short-hand notations $\omega^{2}=\partial_{g} f, \gamma=\partial_{\Delta v} f-\partial_{v} f$, and $\alpha=\partial_{v} f$ have been introduced. They refer to the fact, that equation (4) is a damped, driven, inhomogeneous harmonic oscillator in the net headway, $\ddot{g}+\gamma \dot{g}+\omega^{2} g=F(t)$. The driving term $F(t)$ is given by $F(t)=\alpha V-\alpha v^{*}-$ $\omega^{2} g^{*}-\dot{V}$. Depending on the data, it is suitable to work with the accelerations (if they have been recorded) or with the speeds:

$$
\begin{aligned}
& v(t+\Delta t)=: v^{\prime}=v+\Delta t\left(\omega^{2} g+\gamma \Delta v+\alpha v\right)-\Delta t\left(\omega^{2} g^{*}+\alpha v^{*}\right), \\
& v^{\prime}=(1+\alpha \Delta t) v+\omega^{2} \Delta t g+\gamma \Delta t \Delta v-\Delta t\left(\omega^{2} g^{*}+\alpha v^{*}\right) .
\end{aligned}
$$

For a stable and physically meaningful car-following model, $\omega^{2}>0, \quad \gamma>0$, and $\alpha<0$ should hold at least on average. This model can now being fitted to the car-following episodes of the trajectory data to estimate its parameters. Such an episode is defined by the conditions that at least for more than $30 \mathrm{~s}$ the time headway between lead and following vehicle was between zero and five seconds, and that the average speed during such an episode was larger than $5 \mathrm{~m} / \mathrm{s}$. Altogether 6184 of such episodes have been found in the set of 93 drivers that contained valid data. By assuming that the parameters of this model stay constant at least during one of those car-following episodes, they can be estimated by fitting the model equation to each of the car-following episodes via a non-linear least squares approach. This worked quite well, with a median fitting error of $0.42 \mathrm{~m} / \mathrm{s}$ (the median of the individual root mean squared errors) and a maximum error of $1.83 \mathrm{~m} / \mathrm{s}$. Note, that about 10 $\%$ of the episodes the fitting procedure did not converge, so no parameters could be extracted.

Unfortunately, it is not possible to fit all the terms in equation (5) to the data. This is due to the fact, that only the parameters $a, b, c, d$ of the linear model in equation (6) can be estimated

$$
v^{\prime}=a v+b g+c \Delta v+d .
$$




\begin{tabular}{l|l|l|l} 
Parameter & $\begin{array}{l}\text { Median of } \\
\text { the medians }\end{array}$ & $\begin{array}{l}\text { Width of } \\
\text { the medians }\end{array}$ & $\begin{array}{l}\text { Median of } \\
\text { the widths }\end{array}$ \\
\hline$\alpha[1 / \mathrm{s}]$ & -0.0729 & 0.0575 & 0.206 \\
$\gamma[1 / \mathrm{s}]$ & 0.2960 & 0.1510 & 0.350 \\
$\omega^{2}\left[1 / \mathrm{s}^{2}\right]$ & 0.0208 & 0.0183 & 0.059 \\
$d[\mathrm{~m} / \mathrm{s}]$ & 0.6094 & 0.940 & 2.751 \\
\hline
\end{tabular}

Table 1: Aggregated results of the non-linear least squares fit of the model equations (5), (6) to the car-following episodes.

From this, the physically interesting parameters $\alpha, \gamma, \omega^{2}$ can be determined, it is not possible to compute the individual terms $g^{*}$ and $v^{*}$ of the absolute term (note: $d=\Delta t\left(\omega^{2} g^{*}+\alpha v^{*}\right)$ ). Especially the value of $g^{*}$ would have been of great interest, since it contains directly the sought fluctuation of the preferred headway.

This fitting procedure yields about 60 sets of parameters per driver. From this set of parameters, the median value of each of the parameters and its width (again the interquartile distance is used here) can be computed for each of the drivers. Then, the total median of all the drivers can be computed, and the same can be done for the widths. This yields the results in Table 1.

The values of the medians of the parameters display reasonable numbers. E.g., $\alpha$ should be a small negative number, the size of $\gamma$ is the largest among the interaction parameters $\omega^{2}, \gamma, \alpha$ and indicates that the speed-difference is the most important of all variables that determine human car-following. The small size of $\omega$ is related to the relative unimportance of the distance. However, computing the average period $T=2 \pi / \omega$ from this number yields an oscillation period between $38 \ldots 48 \mathrm{~s}$, which is a good estimate of the oscillation period of the car-following process. This has been verified independently by a Fourier analysis of some of the car-following episodes. The period $T$, as well as the other parameters, might depend on speed, with $T$ increasing with speed, but this has not been investigated.

The most important result is however the behavior of the two widths. The width of the medians of the parameters is a measure of heterogeneity in the drivers, while the median of the (individual) widths is a measure for the fluctuation strength in one driver. It turns out, that driver heterogeneity is by a factor of 2 to 3 smaller than the fluctuation in the parameters of each individual driver. This underlines the results of the analysis of the macroscopic, model-independent data in section 2, which has stated already 


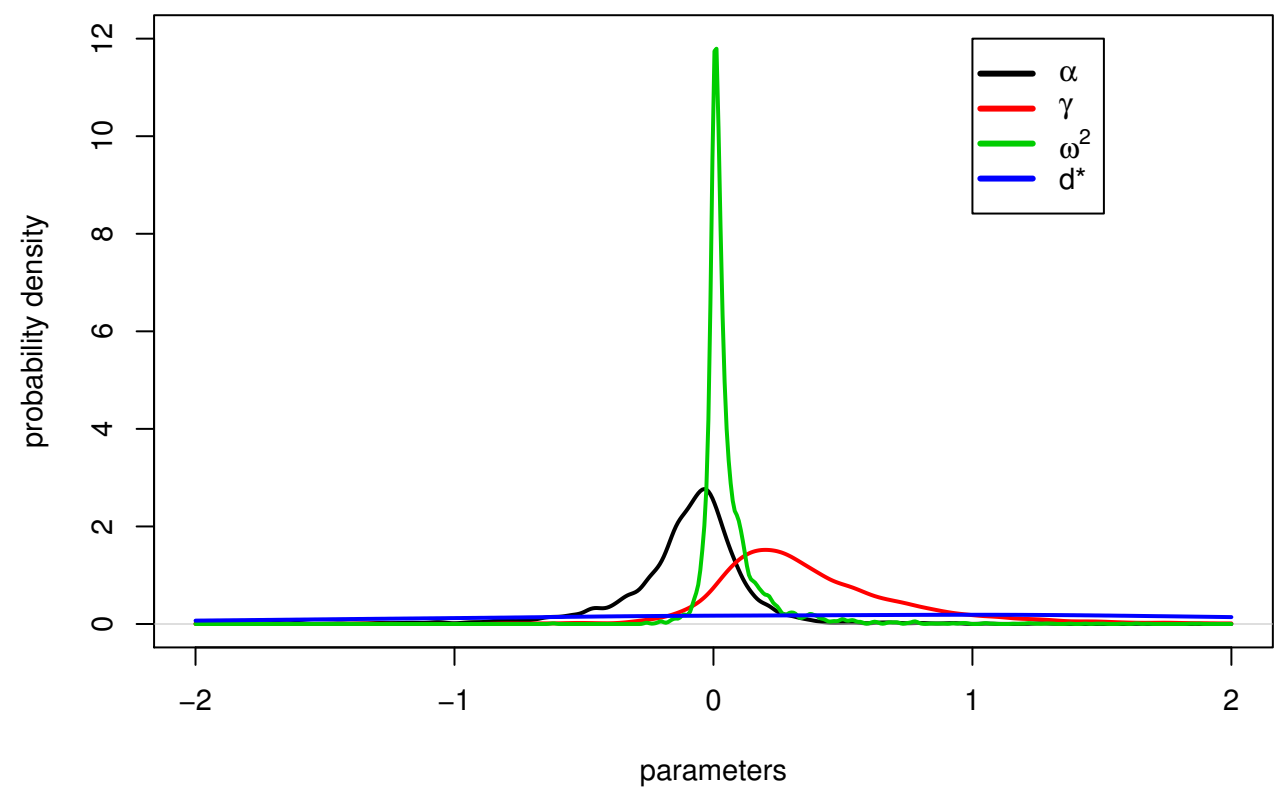

Figure 5: The pdf for all the drivers and all the car-following episodes, and for the four parameters that can be estimated. Since the width of the distribution of $d^{*}$ is large, its density value is very small.

that the main source of the fluctuations in car-following is not the driver heterogeneity but is due to the drivers themselves.

One final consequence can be drawn from Table 1. It could be seen, that the variance of the mean values of the parameters $\alpha, \gamma$, and $\omega$ remains fairly small, while the variance of the absolute term $d^{*}$ is fluctuating much stronger. This can be seen also in Figure 5, where the pdf of all the four parameters for all the car-following episodes is presented. Note, that the parameter $d^{*}$ is related to the preferred headway $g^{*}$ (and the speed $v^{*}$ belonging to this preferred headway) since $d^{*}=\Delta t\left(\omega^{2} g^{*}+\alpha v^{*}\right)$ holds.

Also, for the model (Treiber et al., 2000) used in the appendix to illustrate the behavior described here, the parameters can be computed and fit well with the numbers estimated in Table 1.

Note that strictly speaking, such an analysis is impossible. If the action- 
point model is taken to be serious, then the relationship between the acceleration and the input variables holds only at the action-points itself, and not in the time-intervals following the change of acceleration at the action points. Since between the action points the vehicle is simply following a kinematic rule and not any longer the differential equation (not even the linear one!), there must be an increasing mismatch between the acceleration and the righthand side of the linear model equation. Nevertheless, the method seems to work which is certainly due to the fact that in the data-points following an action-point the mismatch has not grown very large.

Therefore, and because of the fact that the preferred headway cannot be extracted uniquely from $d$, the results obtained can only be called a hypothesis: from the observed stronger fluctuations in the parameter $d$ it will be concluded that it is the preferred headway that is the most important non-constant parameter of the driver.

\section{Conclusions}

This work has presented evidence, that most of the noise in car-following that manifests especially in the large width of the headway distribution is generated already on the level of an individual driver. The basic argument is the approximately equal width of the headway and the speed-difference distribution in SSD-data and trajectory data. If the fluctuations are generated by the heterogeneity of the drivers, then the fluctuations in the SSD-data should be much stronger than the fluctuations in the individual driver data. It has been shown that this is not the case, both fluctuations are of the same size.

To explain this, a hypothesis has been formulated which claims that the fluctuations can be traced back to a permanent change of the drivers preferred time headway in car-following. Since the preferred headway cannot be measured directly, a rather indirect and somewhat speculative model-based approach was used that gives some support to this claim. In addition, there is at least one approach (Tordeux et al., 2010), where researchers have assumed that time headways will be adopted to the current situation, however not as variable as it is assumed here.

Nevertheless, the whole analysis demonstrated here shows that car-following models should be put onto another basis if they want to describe the human car-following behavior correctly; where correctly means, that not only the mean values should be described (which seems to work already quite well), 
but also the fluctuations around these mean values. A simple approach that follows immediately from the analysis above is to use any of the already existing car-following models and enhance them with a mechanism that causes the preferred distance to fluctuate. An additional step would be to add an action-point mechanism. Both approaches are demonstrated explicitly with an existing model in the appendix. Still, the original model upon which such an enhancement is to be added does not need to be changed at all. However, it seems that most of the details usually present in microscopic traffic flow models are not needed since the above mentioned linear model is able to describe car-following data with an average error of around $0.4 \mathrm{~m} / \mathrm{s}$.

\section{Appendix A. A numerical example}

To show the general idea, the car-following model in (Treiber et al., 2000) together with an acceleration noise term (which is not present in the original formulation of the model) will be used:

$$
\dot{v}=a\left(1-\left(\frac{v}{v_{\max }}\right)^{4}-\left(\frac{g_{0}+v T-\frac{v \Delta v}{2 \sqrt{a b}}}{g}\right)^{2}\right)+\sigma_{a} \xi .
$$

Here, $a, \quad \sigma_{a}$ represent the preferred acceleration and the acceleration noise, respectively, the noise is represented by a random number in the interval $[-0.5,0.5]$. The speed is constrained to the range $\left[0, v_{\max }\right]$, the parameters $g_{0}, b, T$ are the distance to the vehicle in front when standing, the preferred deceleration, and the preferred net time headway, respectively. The simulation study below uses reasonable values $a=1.5 \mathrm{~m} / \mathrm{s}^{2}, \sigma_{a}=0.4 \mathrm{~m} / \mathrm{s}^{2}, T=1.5$ $\mathrm{s}, b=4.5 \mathrm{~m} / \mathrm{s}^{2}, v_{\max }=40 \mathrm{~m} / \mathrm{s}$, and $g_{0}=3 \mathrm{~m}$. The simulation takes a string of 20 vehicles that follow a lead vehicle driving with constant speed $(25 \mathrm{~m} / \mathrm{s})$. The resulting set of differential equations is solved with an Euler scheme of time step size $\Delta t=0.1 \mathrm{~s}$ as in the data-analysis above. Only the trajectory of the last vehicle is analyzed in the following, the last vehicle is used to catch eventual string instabilities.

Three versions of this model have been considered: the basic one as described so far (named "noisy IDM" in Figure A.6), a version with an actionpoint mechanism (named "AP" in Figure A.6), and a model were in addition the preferred time headway $T$ fluctuates (named "noisy T" in Figure A.6).

The action-point mechanism is implemented as described in (Wagner, 2011): either at random or when the vehicle is in imminent danger of crash- 
ing into the vehicle in front an action-point is triggered which changes the acceleration of the vehicle to a new value as computed from equation (A.1). The random component is modeled by deciding with a probability 0.2 in any time-step of $0.1 \mathrm{~s}$ duration whether or not an action-point should be issued. This gives an average time between two action-points of $0.5 \mathrm{~s}$, roughly in line with the empirical analysis above. The test for danger is implemented by the comparison of the current acceleration with another safe acceleration. This safe acceleration is computed by inserting in equation (A.1) a much shorter headway, e.g. the reaction time $T_{r}=0.5 \mathrm{~s}$. If the current acceleration is by chance larger than the safe acceleration, an action-point is triggered, too, and the current acceleration is set to this safe acceleration. This is checked for in any time-step, to avoid crashing with the lead vehicle because of the action-point mechanism. (This is needed, since the time between two actionpoints can become very long, leading to crashes in an otherwise crash-free model.) Between two action-points, the vehicle simply follows its kinematic trajectory:

$$
x(t)=x_{i}+v_{i}\left(t-t_{i}\right)+\frac{1}{2} a_{i}\left(t-t_{i}\right)^{2}, t \in\left[t_{i}, t_{i+1}[\right.
$$

where $x_{i}, v_{i}, a_{i}$ are defined as the position, speed, and acceleration at time $t_{i}$, respectively. The new acceleration $a_{i}$ is computed by inserting the current values $g_{i}, v_{i}, \Delta v_{i}$ into equation (A.1). A typical result is displayed in Figure A.6 by plotting the trajectories in the speed-difference-distance space. It demonstrates, that at least for this model both acceleration noise alone, as well as the action-point mechanism is not sufficient to produce the observed fluctuations in the time headway.

The change in the preferred headway is modeled as follows. Whenever an action-point is triggered, a new preferred headway is generated, too. This is done by drawing a random number in the interval $\left[T_{1}, T_{2}\right]$, where $T_{1}=1 \mathrm{~s}$ and $T_{2}=2 \mathrm{~s}$. For a more realistic modeling, the preferred headways should be drawn from another distribution, like e.g. a shifted Gamma distribution. Even an explicit stochastic equation can be used as has been suggested in (Wagner, 2004). Furthermore, the whole model can be made simpler: with a stochastic headway, there is no need for the acceleration noise. When setting $\sigma_{a}$ to zero, the trajectory produced looks very similar to the one displayed in Figure A.6. 


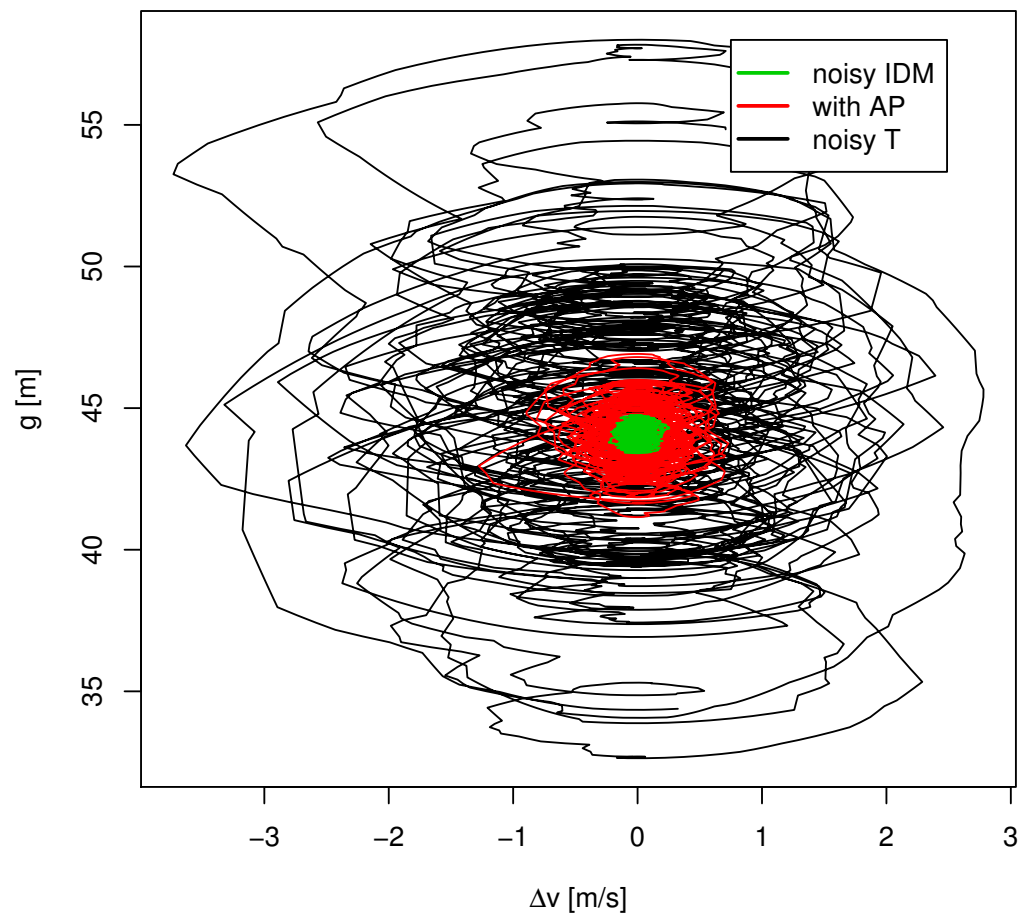

Figure A.6: Trajectories of the three different model variants described in the text.

\section{References}

Alexiadis, V., Colyar, J., Halkias, J., Hranac, R., McHale, G., August 2004. The next generation simulation program. ITE Journal 74 (8), $22-26$.

Cowan, R. J., 1976. Useful headway models. Transportation Research 9 (6), $371-375$.

Fancher, P., Ervin, R., Sayer, J., Hagan, M., Bogard, S., Bareket, Z., Mefford, M., Haugen, J., 1998. Intelligent Cruise Control Field Operational Test. U.S. DOT, HS 808849 Edition, University of Michigan Transportation Research Institute.

Flötteröd, G., Wagner, P., Wang, Y.-P., 2011. Identifiability and practical 
relevance of complex car-following models. In: Ninth International Conference on Traffic And Granular Flow, 2011. pp. 37-45.

Gipps, P., 1981. A behavioural car-following model for computer simulation. Transportation Research Part B 15 (2), 105-111.

Helly, W., 1959. Simulation of bottlenecks in single lane traffic flow. In: Proceedings of the symposium on theory of traffic flow. pp. 207-238.

Hoogendoorn, S., Hoogendoorn, R. G., Daamen, W., 2012. Wiedemann revisited - new trajectory filtering technique and its implications for carfollowing modeling. Transportation Research Record 2260, 152-162.

Hoogendoorn, S. P., Bovy, P., 1998. A new estimation technique for vehicletype specific headway distributions. Transportation Research Record 1646, $18-28$.

Kerner, B. S., 2004. The Physics of Traffic. Springer.

Kesting, A., Treiber, M., 2009. Calibration of car-following models using floating car data. In: Appert-Rolland, C., Chevoir, F., Gondret, P., Lassarre, S., Lebacque, J.-P., Schreckenberg, M. (Eds.), Traffic and Granular Flow '07. Springer Berlin Heidelberg, pp. 117-127.

Knospe, W., Santen, L., Schadschneider, A., Schreckenberg, M., 2002. Singlevehicle data of highway traffic: Microscopic description of traffic phases. Physical Review E 65 (5), 056133.

Krauss, S., Wagner, P., Gawron, C., 1997. Metastable states in a microscopic model of traffic flow. Physical Review E 55 (5), 5597-5602.

Krbalek, M., Helbing, D., 2004. Determination of interaction potentials in freeway traffic from steady-state statistics. Physica A: Statistical Mechanics and its Applications 333 (1-4), 370-378.

Krbalek, M., Šeba, P., Wagner, P., 2001. Headways in traffic flow: Remarks from a physical perspective. Physical Review E 64 (6), 066119.

May, A. D., 1990. Traffic Flow Fundamentals. Prentice Hall, Englewood Cliffs, NJ. 
Newell, G., 2002. A simplified car-following theory: a lower order model. Transportation Research Part B 36 (3), 195-205.

Ossen, S., Hoogendoorn, S. P., Gorte, B., 2007. Inter-driver differences in car-following: A vehicle trajectory based study. Transportation Research Records 1965, 121-129.

Petty, K., 1995. Freeway Service Patrol Project and the I-880 database. Tech. rep., University of Berkeley, California, http://ipa.eecs.berkeley.edu/ pettyk/FSP/.

Todosiev, E. P., Barbosa, L. C., 1963/64. A proposed model for the drivervehicle-system. Traffic Engineering 34, 17-20.

Tordeux, A., Lassarre, S., Roussignol, M., 2010. An adaptive time gap carfollowing model. Transportation Research Part B 44 (8-9), 1115-1131.

Treiber, M., Hennecke, A., Helbing, D., 2000. Congested traffic states in empirical observations and microscopic simulations. Physical Review E $62(2), 1805-1824$.

Wagner, P., 2004. Modelling traffic flow fluctuations. eprint arXiv:condmat/0411066.

Wagner, P., 2005. Empirical description of car-following. In: Hoogendoorn, S. P., Luding, S., Bovy, P. H. L. (Eds.), Traffic and Granular Flow '03. Springer, pp. 15-28.

Wagner, P., 2006. How human drivers control their vehicle. European Physical Journal B 52 (3), 427-431.

Wagner, P., 2011. A time-discrete harmonic oscillator model of human carfollowing. European Physical Journal B 84 (4), 713-718.

Wiedemann, R., 1974. Simulation des Straßenverkehrsflußes. Tech. rep., Institut für Verkehrswesen, Universität Karlsruhe, Heft 8 der Schriftenreihe des IfV, in German. 